\title{
Numerical study of a cosmological relaxation model of the Higgs boson mass
}

\author{
Marco Michelø* \\ Max-Planck-Institut für Physik, Föhringer Ring 6, 80805 München, Germany \\ and Arnold Sommerfeld Center, Ludwig-Maximilians-Universität, \\ Theresienstraße 37, 80333 München, Germany
}

(Received 27 November 2019; accepted 12 May 2020; published 3 June 2020)

\begin{abstract}
In light of no new physics being discovered at the LHC, ideas which tackle the hierarchy problem without novelties around the TeV scale must be taken seriously. Such is a cosmological relaxation model of the Higgs boson mass, proposed in the pre-LHC era, which does not rely on new physics below the Planck scale. This scenario introduces a different notion of naturalness according to which the vacuum with a small expectation value of the Higgs field corresponds to an infinitely enhanced entropy point of the vacuum landscape that becomes an attractor of cosmological inflationary evolution. In this framework we study numerically the evolution of the Higgs vacuum expectation value (VEV). We model the inflationary vacuum-to-vacuum transitions that are triggered by nucleation of branes charged under three-form fields as a random walk. In particular, we investigate the impact of the number of coupled three-forms on the convergence rate of the Higgs VEV. We discover an enhanced rate when increasing the number of brane charges. Moreover, we show that for late times the inclusion of more charges is equivalent to additional brane nucleations.
\end{abstract}

DOI: 10.1103/PhysRevD.101.115007

\section{INTRODUCTION}

Rooted in the quadratic sensitivity of the Higgs boson mass term to the UV cutoff, the hierarchy problem is still an unresolved puzzle in theoretical physics. (For a discussion of the hierarchy problem and its connection to naturalness, see [1].) Naturally, since the standard picture raised the problem in the first place a solution to it most likely has to contain new ingredients. Most of the proposed solutions such as supersymmetry, which has no quadratic divergences [2], technicolor [3], extra dimensions [4,5] or large number of species [6], predict stabilizing new physics around a scale not much larger than the weak scale. However, these predicted deviations from the standard model have not been observed at currently available energy scales. Nonetheless, in the pre-LHC era an alternative idea of solving the hierarchy problem via cosmological relaxation of the Higgs boson mass has been suggested $[7,8]$. This scenario does not rely on any low energy physics as it can push the scale of the onset of new physics up to the Planck scale. It also introduces a notion of naturalness [1] that is fundamentally different from the standard one by 't Hooft [9]. Here, the vacuum with the small value of the

\footnotetext{
michel@mpp.mpg.de
}

Published by the American Physical Society under the terms of the Creative Commons Attribution 4.0 International license. Further distribution of this work must maintain attribution to the author(s) and the published article's title, journal citation, and DOI. Funded by SCOAP .
Higgs boson mass has infinite entropy and represents an attractor of cosmological evolution. The nonobservation of any new physics at the LHC gives serious motivation for a detailed study of this scenario. A list of recent references based on the same idea can be found in [1].

In this paper we first confirm the relaxation of the Higgs vacuum expectation value (VEV) to the attractor value by numerical simulations. Furthermore, we extend the analysis to additional three-form fields and study their effect on the convergence rate. This is motivated by embedding the attractor scenario in various fundamental theories that contain multiple forms.

The paper is structured as follows. First, we briefly review the cosmic attractor model in Sec. II. In Sec. III, we map a simplified model to a stochastic framework. The results and interpretation of the numerical simulations are provided in Sec. IV. We conclude the discussion in Sec. V.

\section{COSMIC Higgs VEV RELAXATION MECHANISM}

In this section we briefly review the cosmic attractor model introduced in [7] and further refined in [8,1]. This model exhibits a high degeneracy of vacua around a certain hierarchically small value of the Higgs VEV $\Phi_{*}$. This point of enhanced entropy is reached via brane nucleations during inflationary cosmological evolution. Since inflation lasts eternally $[10,11]$, the system has infinitely available time to converge to the attractor point. It is therefore natural to find oneself in the vacuum with maximal entropy and the corresponding Higgs VEV $\Phi_{*}$. 
The crux of the mechanism is to couple the Higgs to a massless three-form field which is sourced by a two-brane with charge $Q$ set by the Higgs field itself. The fundamental nature of the brane is not important; in particular, as shown in $[7,8]$, it can be resolved in the form of a domain wall of a heavy axion. Crossing the two-brane (or axionic domain wall) leads to a jump in the field strength $F$ with the distance set by the brane charge:

$$
\Delta F=Q(\Phi) .
$$

On the other hand, $F$ is backcontrolling the vacuum expectation value of the Higgs field via

$$
\Phi^{2}=\frac{1}{\lambda}\left(\frac{F^{2}}{M^{2}}-m^{2}\right),
$$

where $\lambda$ is a coupling constant, $M$ is some cutoff, and $m$ incorporates all other contributions to the effective Higgs boson mass. Using Eqs. (1) and (2), the change of the Higgs VEV for small values of $Q$ when crossing a two-brane is

$$
\Phi \Delta \Phi=-\frac{F}{M^{2} \lambda} Q+\mathcal{O}\left(Q^{2}\right) .
$$

The final ingredient is the exact form of the dependence of $Q$ on $\Phi$. With the effective brane charge given by

$$
Q(\Phi)= \pm \frac{\left(\Phi^{N}-\Phi_{*}^{N}\right)^{K}}{M^{N K-2}},
$$

where $N$ and $K$ are positive and integer valued parameters, the sign of the charge is not fixed, and the difference in the Higgs VEV for neighboring vacua vanishes for $\Phi \rightarrow \Phi_{*}$. Correspondingly, the density of vacua diverges at that point. The result is a probability distribution for $\Phi$ with a singular peak around $\Phi_{*}$. This point is called an attractor since, given infinite time, $\Phi$ will inevitably move arbitrarily close to $\Phi_{*}$.

For derivation of Eqs. (1)-(4) and further details, we refer to the original papers $[1,7,8]$. In the following sections the terms brane nucleation and time step are used interchangeably, and $\Phi$ and $\lambda$ are measured in units of Planck mass $M_{P}$.

\section{RANDOM WALK MODEL}

Since we are solely interested in the efficiency of the attractor mechanism, we neglect all contribution to the Higgs boson mass other than from $F$, so we set $m=0$. Thus, the change of $\Phi$ simplifies to

$$
\Delta \Phi=\frac{Q}{\lambda M^{2}}= \pm \frac{\left(\Phi^{N}-\Phi_{*}^{N}\right)^{K}}{\lambda M^{N K}} .
$$

Sufficiently close to the attractor, backreaction on the inflationary background can be ignored; therefore, the probability of nucleating a brane or an antibrane can be assumed to be equal. Correspondingly, we assign the probability $P=0.5$ to moving in either positive or negative directions.

In the following we will therefore model the time evolution of $\Phi$ as a symmetric random walk. This is an extremely good approximation. Because brane nucleation is a rare and nonperturbative process, the nucleation of subsequent branes in the new vacuum is not sensitive to the original direction of nucleation. In other words, by the time a given nucleation happens, the walls of the bubble from the previous nucleation are exponentially far away and do not affect the succeeding nucleation direction.

We further simplify the original expressions by assuming $\Phi_{*}=0$, merging the coupling constant $\lambda$ and the cutoff $M$ into one parameter $\mu$ and keeping the exponent of this parameter independent of $\nu \equiv N K$. The change $\Delta \Phi$ at each discrete time step (or equivalently brane nucleation) is then given in compact form by

$$
\Delta \Phi(\tilde{\Phi}, \mu, \nu)=\left\{\begin{array}{ll}
+\mu \tilde{\Phi}^{\nu} & P=0.5 \\
-\mu \tilde{\Phi}^{\nu} & P=0.5
\end{array},\right.
$$

where $P$ denotes the probability of the specific outcome, and $\tilde{\Phi}$ is the current value of the variable $\Phi$. For the remaining free parameters, we assume $\mu \in(0,1)$ and $\nu$ is an integer and greater than one.

Thus, the random walk can be defined iteratively by

$$
\Phi_{i+1}=\Phi_{i}+\Delta \Phi\left(\Phi_{i}, \mu, \nu\right),
$$

where $i$ indexes the brane nucleations, and $\Phi_{i}$ denotes the value of $\Phi$ after the $i$ th brane has nucleated.

It is straightforward to generalize this model to multiple different charges on the brane. Since these are independent from each other, the sequence in Eq. (7) can be generalized to

$$
\Phi_{i+1}=\Phi_{i}+\sum_{k=1}^{d} \Delta \Phi\left(\Phi_{i}, \mu_{k}, \nu_{k}\right),
$$

for $d$ distinct charges and possibly different and independent parameters $\nu_{k}$ and $\lambda_{k}$. So at every time step, the VEV of the Higgs is shifted by $d$ different terms where, again, the sign of each contribution is equally likely due to the unfixed signs of the individual charges.

\section{SIMULATIONS}

The random walk defined by Eq. (8) can be simulated on a computer. Of course, due to the statistical nature of the process, quantitative analyses can only be performed with a high number of realizations. Here, we are interested in how the number of distinct brane charges $d$ affects the convergence rate of the random walk. 


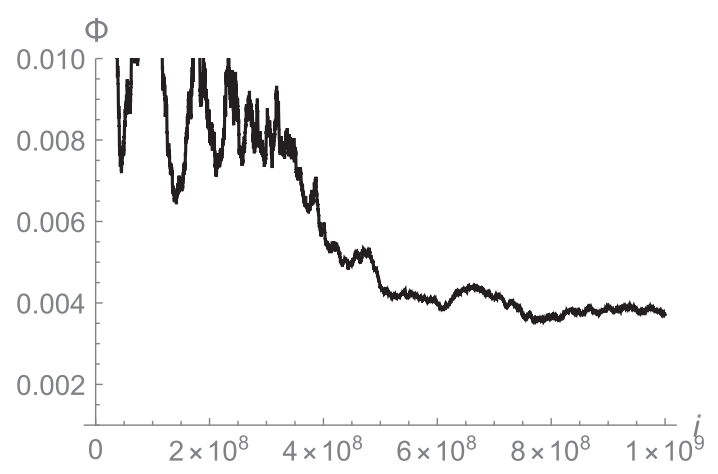

(a) $d=1$

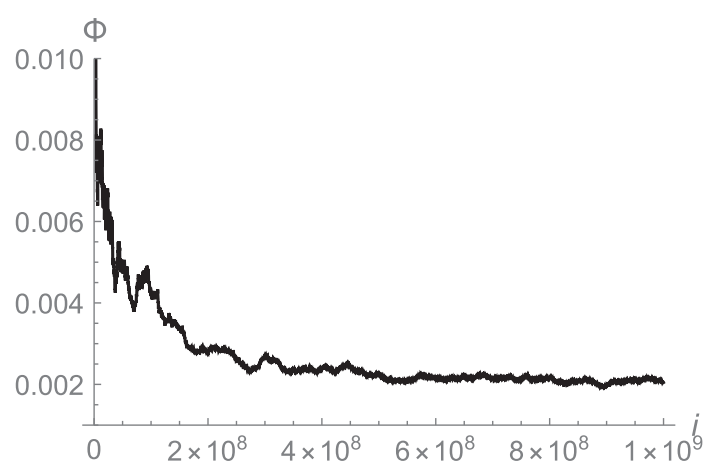

(b) $d=10$

FIG. 1. Exemplary realizations of the random walk defined in Eq. (8) with $\mu=0.5, \nu=3$, and $\Phi_{0}=0.1$ for different numbers of brane charges. The plot range has been set to emphasize late time behavior. In this specific example the run with the higher number of charges converges faster to smaller values compared to the $d=1$ case.

All simulations in this section have been performed with an initial value of the Higgs VEV given by $\phi_{0}=0.1$, and the two free parameters were set to $\lambda=0.5$ and $\nu=3$, respectively, and equal for all three-form fields. Every simulation was calculated for $n=10^{9}$ steps. For late times we plot illustrative random walks for $d=1$ and $d=10$ in Fig. 1. Matching the intuitive picture, both walks decrease on average as a function of the time step $i$. Of course, the smaller $\Phi$ becomes, the slower the rate becomes in accordance with Eq. (6). Thus, for the Higgs VEV to relax infinitely close to zero, infinite time would be required. However, this time requirement can be accounted for by eternal inflation [10,11], as already explained in Sec. II.

Next, we analyze the dependence of the convergence rate of $\Phi$ on the number of charges $d$. For this purpose, we

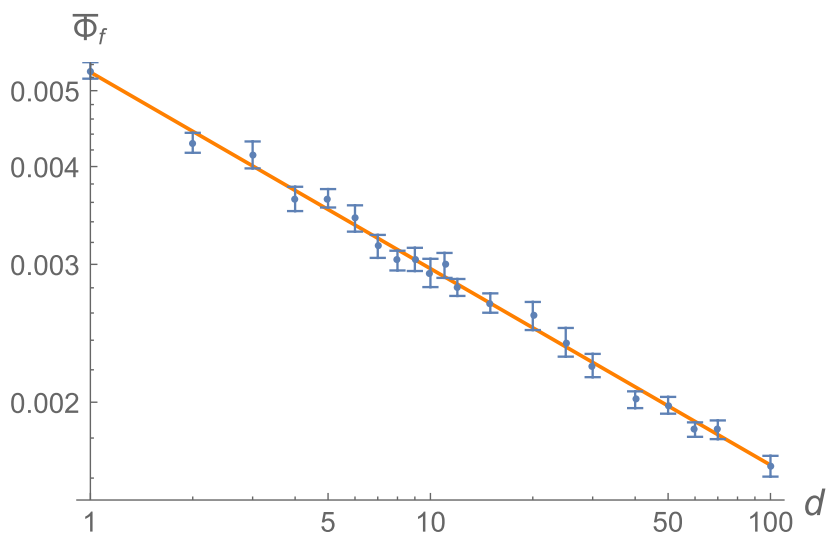

FIG. 2. Average final value $\bar{\Phi}_{f}$ after $n=10^{9}$ time steps as a function of the number of charges $d$. For every point the average has been taken over 100 runs. For a fit function of the form $a \cdot x^{b}$, the parameters attain the following values: $a=0.0053$ and $b=$ -0.25 with standard errors $\sigma_{a}=5.3 \times 10^{-5}$ and $\sigma_{b}=0.005$. The parameters of model (8) have been set to $\mu=0.5$ and $\nu=3$ with an initial value given by $\Phi_{0}=0.1$. The standard error is depicted as error bars around its mean value. average over the final value $\Phi_{f}$ after $n=10^{9}$ steps for 100 runs $^{1}$ for various $d \in[1,100]$. The corresponding data are plotted in Fig. 2 in blue. In accordance with Fig. 1, we clearly observe an enhanced relaxation efficiency for higher $d$.

For quantitative conclusions, we fit the data in Fig. 2 with a function of the form $a \cdot x^{b}$ in orange. The fit parameters and their respective standard deviation are presented in Table I. The exponent matches, within statistical errors, the averaged exponent when fitting the values of $\Phi$ for individual runs. This completely matches the analytic intuition that increasing the brane nucleation channels is equivalent (up to a numerical factor) to the nucleation of more branes. The only difference that can be observed is at the beginning when $\Phi$ is still large and when the attractor's pull is the strongest. Greater $d$ results in larger jumps within $n^{\prime}$ steps in comparison with an equivalent run with $n^{\prime \prime}=d \cdot n^{\prime}$ steps and only one charge. For later times and small $\Phi$, however, the difference between jump distances for neighboring values of $\Phi$ becomes negligible.

We also repeated the analysis for $\nu \in\{2,4\} .^{2}$ The results are presented in Table I. Again, we observe that the inclusion of more brane charges is, up to a numerical factor, equivalent to additional nucleation of branes. We also checked for $\nu=3$ that varying $\mu \in[0.05,0.5]$ only results in a change of the prefactor $a$. We expect this behavior to also hold for smaller values of $\mu$.

\footnotetext{
${ }^{1}$ Runs that diverged at some point $\left(\Phi_{i}>1\right.$ for some $\left.i\right)$ were discarded and reset.

${ }^{2}$ Within the considered number of steps, the numerical value of $\Delta \Phi$ with $\nu \geq 5$ cannot be resolved accurately enough with standard machine precision. However, in principle this problem can be solved, and we do not expect any significant deviation from our results in this regime.
} 
TABLE I. Fitting values for the evolution of $\Phi$ as a function of brane charges and number of nucleated branes. The values for the ladder are marked by a tilde. For both cases the fitting function is of the form $a \cdot x^{b}$. The standard errors are denoted by $\sigma$.

\begin{tabular}{ccccccccc}
\hline \hline$\nu$ & $a$ & $\sigma_{a}$ & $b$ & $\sigma_{b}$ & $\tilde{a}$ & $\sigma_{\tilde{a}}$ & $\tilde{b}$ & $\sigma_{\tilde{b}}$ \\
\hline 2 & $4.8 \times 10^{-5}$ & $1.1 \times 10^{-6}$ & -0.48 & 0.014 & 0.05 & 0.02 & -0.5 & 0.04 \\
3 & 0.0053 & $5.3 \times 10^{-5}$ & -0.25 & 0.005 & 0.17 & 0.07 & -0.25 & 0.06 \\
4 & 0.026 & $2 \times 10^{-4}$ & -0.17 & $2.7 \times 10^{-3}$ & 0.25 & 0.1 & -0.16 & 0.04 \\
\hline \hline
\end{tabular}

\section{CONCLUSIONS}

In this paper we have studied numerically the model first introduced in [7,8], which solves the hierarchy problem by cosmological relaxation of the Higgs boson mass towards the attractor vacuum during eternal inflation. In this scenario the Higgs boson mass/VEV is changed due to nucleation of branes (or axionic domain walls). At the same time the Higgs VEV acts as a backcontrol parameter that directs the convergence of the relaxation progress.

Such an attractor could, in principle, be realized in various different models, each with its own specifics. In this analysis, however, we only focused on universal key features of the cosmic attractor mechanism. For this we have modeled the Higgs VEV evolution as a random walk with each step mimicking a vacuum transition triggered by a brane nucleation. The observed convergence to $\Phi_{*}=0$, which represents our attractor point, is in accordance with analytic considerations. We then generalized this stochastic model to multiple different charges sourced by a two-brane and studied the impact of their number on the relaxation rate of the Higgs VEV. We showed that a higher number of three-form fields leads to a faster convergence rate. That is, less brane nucleations are necessary to relax the Higgs vacuum expectation value below a given value. This confirms the intuitive picture that adding brane charges is equivalent to an increase of brane nucleation channels.

\section{ACKNOWLEDGMENTS}

We thank Gia Dvali for valuable discussions throughout the project and helpful comments on the manuscript. We are also very grateful to Georgios Karananas for insightful discussions.
[1] G. Dvali, Cosmological relaxation of Higgs mass before and after LHC and naturalness, arXiv:1908.05984.

[2] H. P. Nilles, Supersymmetry, supergravity and particle physics, Phys. Rep. 110, 1 (1984).

[3] S. Weinberg, Implications of dynamical symmetry breaking, Phys. Rev. D 13, 974 (1976); Addendum, Phys. Rev. D 19, 1277 (1979); L. Susskind, Dynamics of spontaneous symmetry breaking in the Weinberg-Salam theory, Phys. Rev. D 20, 2619 (1979).

[4] N. Arkani-Hamed, S. Dimopoulos, and G. R. Dvali, The hierarchy problem and new dimensions at a millimeter, Phys. Lett. B 429, 263 (1998).

[5] L. Randall and R. Sundrum, A Large Mass Hierarchy from a Small Extra Dimension, Phys. Rev. Lett. 83, 3370 (1999).

[6] G. Dvali, Black holes and large N species solution to the hierarchy problem, Fortschr. Phys. 58, 528 (2010); G. Dvali and M. Redi, Phenomenology of $10^{32}$ dark sectors, Phys. Rev. D 80, 055001 (2009); N. Arkani-Hamed, T. Cohen, R. T. D'Agnolo, A. Hook, H. D. Kim, and D. Pinner, Solving the Hierarchy Problem at Reheating with a Large Number of Degrees of Freedom, Phys. Rev. Lett. 117, 251801 (2016).

[7] G. Dvali and A. Vilenkin, Cosmic attractors and gauge hierarchy, Phys. Rev. D 70, 063501 (2004).

[8] G. Dvali, Large hierarchies from attractor vacua, Phys. Rev. D 74, 025018 (2006).

[9] G. 't Hooft, Naturalness, chiral symmetry, and spontaneous chiral symmetry breaking, NATO Sci. Ser. B 59, 135 (1980).

[10] A. Vilenkin, The birth of inflationary universes, Phys. Rev. D 27, 2848 (1983).

[11] A. D. Linde, Eternally existing self-reproducing chaotic inflationary Universe, Phys. Lett. B 175, 395 (1986). 